\title{
Analysis on the Application of the virtual reality Technology in the Graphic Design
}

\author{
HUANG Wei-wen
}

\author{
Media Art College, Chongqing College of Electronic Engineering, Chongqing 401331, China \\ huangweiwen123@163.com
}

Keywords: Virtual reality technology; Graphic design; Digital

\begin{abstract}
With the introduction of The virtual reality technology, it not only changes the means of graphic design, more promote the leap of the graphic design thought. Although The virtual reality technology applied in society is not very long, and in some way also has certain imperfect, but it has the advantage of making that it is considered to be the main mode of human-computer interaction in the future. The application of The virtual reality technology in graphic design, promoted the digital process of the graphic design, The virtual reality technology whether in function or other information stored on computer models with reality are totally close, this similarity makes The virtual reality technology in the future occupy the dominant position in the graphic design. This article mainly discusses the application of The virtual reality technology in graphic design.
\end{abstract}

\section{Introduction}

The virtual reality system from the angle of the user-oriented is mainly from the following three parts: the virtual scene, virtual avatar, human-computer interaction interface network. As a The virtual reality system simulation of real world and virtual scene is the key part of The virtual reality system, thus generated virtual scene technology is especially important. Scene rendering technology is the foundation of The virtual reality technology and the core. It including the purpose of gain real 3D scene geometry data, and based on the needs of the application, the 3D data obtained using virtual scene is established.

\section{The Definition of The virtual reality technology}

VR (The virtual reality, VR), also known as dreamland, is using the computer simulation to create a 3D Virtual world, to provide users simulation about senses such as vision, hearing, touch, let users as immersive, timely, there is no limit to observe things in three dimensions. VR technology the application in the field of aerospace, concentrated in the U.S. military simulation training of pilots, pilots in flight simulator as if on a real graphic, operating various kinds of instrument equipment on the screen, visual can generate real-time scene outside the cockpit window, such as the airport and runway, buildings, rivers, clouds, etc.

The main characteristic of The virtual reality technology: (1) In simple terms is to make people have an immersive feeling, that is to say, this technology can make people not only into the virtual world from the visual and auditory, but let a person in the form of a whole into The virtual reality system, make people from vital reality, really use your body to perceptual knowledge and thought processes. (2) Interactive. This feature is mainly manifested in The virtual reality and the traditional CAD model and the difference between the three dimensional animation, The virtual reality technology to create is an interactive and open environment, this environment related monitoring equipment can be used to impact on the user or by the user. That is to say, the user can make use of the relevant equipment to control the objects in the virtual environment, realize the user operability of virtual environment, the corresponding object can also be through their own behavior to influence the virtual environment, the extent of the computer system based on virtual environment changes, to adjust relevant virtual environment and change. 


\section{The Key Technology of the virtual reality}

The virtual reality is a five-dimensional space-time on real world simulation technology. Five-dimensional space-time is to point to in addition to the normal three-dimensional space, also includes one dimensional time and space and natural interactive simulation, thus to view a The virtual reality system is the key to whether mature standard 3D virtual space environment and natural interaction is cohesion. The virtual reality system is mainly composed of 3D graphics generation technology, dynamic environment modeling and stereo display and sensor technology, system integration technology and application system development tools of five parts. The 3D graphics generation technology is mainly aimed at the image generated in real time; Dynamic environment modeling technology is based on the actual demand to obtain the corresponding 3D data, and based on 3D data and actual environment the same virtual environment model; Stereo display and sensor technology is to improve reality and a key technology of virtual exchange. Users through The virtual reality software can truly feel the man-made scene in virtual environment, and with the aid of computer and external equipment to realize the human-computer interaction.

The Application of the virtual reality technology in the Graphic design

The virtual reality in urban planning urban planning applications map of city planning map of urban planning has been the demand for new visualization technology is one of the most urgent field, virtual reality technology can be widely used in urban planning, and bring real and substantial benefits: Show immersive and interactive planning scheme of virtual reality system not only can bring a strong, vivid sensory impact to users, get the immersive experience, can always get project data in a virtual environment in real time through the data interface, convenient for large and complex project planning, design, tendering, approval, management, is conducive to the design and management of design and scheme of auxiliary review of the various planning and design. Designed to avoid the risk of. Virtual reality virtual environment is established by the combination of digital model based on real data to establish and become, strictly follow the project design standards and requirements for the establishment of realistic 3D scenes, reproducing the real planning projects "'. Speed design using virtual reality system, we can easily be modified, change the height of the building, changing the building exterior materials, change color, green density, as long as the changes in the system parameters can be. Thus greatly accelerating the speed and quality of design, improve the efficiency of design and modification, but also save a lot of funds, provide cooperation platform. Virtual reality technology will enable the planning department, project developers, engineers and the public from any angle, real-time interactive planning to see real results, to better grasp the intent of the design patterns and understand the city planner. Effective cooperation is the premise to ensure the ultimate success of city planning, virtual reality technology for such cooperation provides the ideal bridge, which is the traditional means such as plan, effect graph, sand table and animation can not reach.
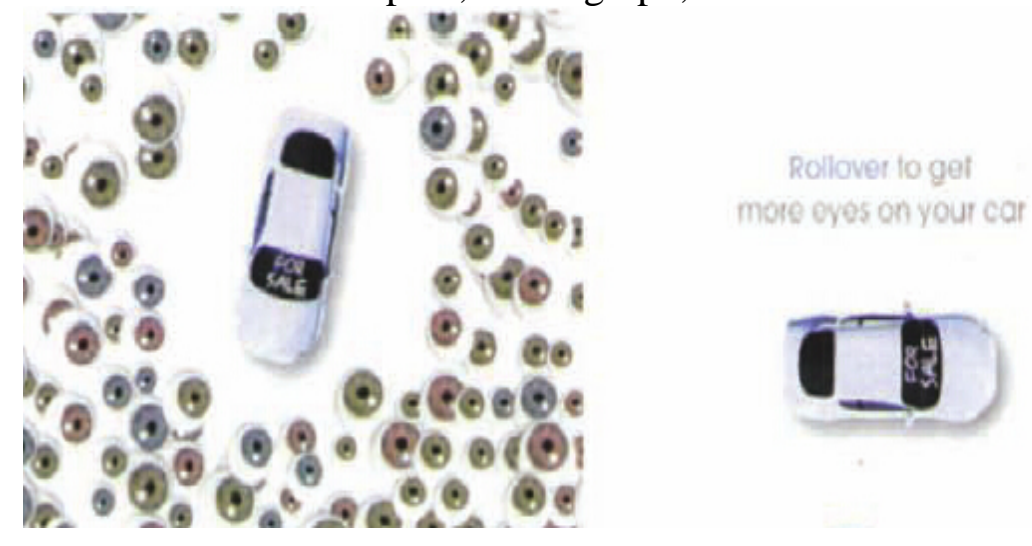

Fig.1: Used car network multimedia interactive advertising

Graphic design is directly created visual graphic elements design activity, from abstract form in the real shape and color of visual symbolic language creation process again. With real existence "instead 
of" 2D printed graphic elements, embodies the graphic elements from two-dimensional space to the expansion of the real space, in order to give the viewer a livelier, strong mental and visual impact. For "no littering" outdoor advertising, in figure 2 of this light boxes designed on the station became transparent bins, every day in the garbage will be found on the station concentrated here, and along with the growth of the time is piling up. In this case, the author changed his graphic symbol for the real garbage, to enhance the visual appeal.
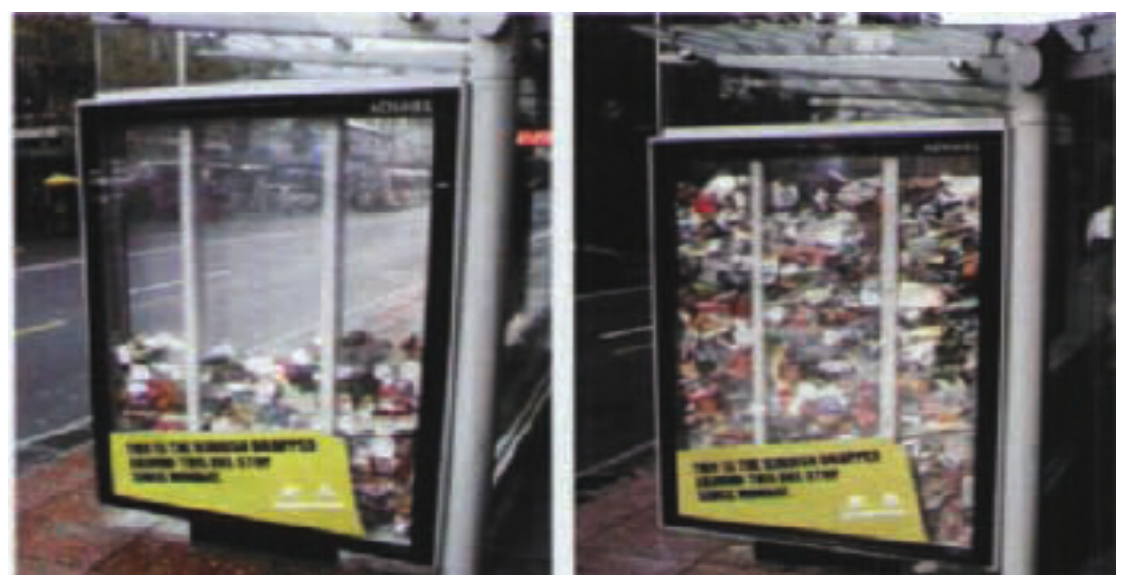

Fig. 2 Transparent bin

Objects in space must produce projection, a projected pattern is closely related to produce projection graphics, many designers have found the projection of the virtual space form, and by using the projection made many successful design cases. Also reflected the graphic design elements of the use of visual should perform multidimensional. Medium is the carrier of information, is to spread the factors in the process of mutual coupling of ties, including with other elements of the floorboard of the media tools. Rely on the media of visual elements change diverse applications, namely the method of using the change of media for design innovation also reflected the visual elements should "multidimensional" performance. Modeling of various properties characteristics of media, such as structure, material, color, etc in physical state properties, tries to use for designers, creative inspiration. The modeling of medium characteristics can not only take advantage, you can even change, combining with the design of the modeling of medium form or modification processing, display, also can achieve the result that find everything new and fresh. Figure 3 is for wildlife protection poster design works of posters, designers will showcase compared to held the cage of wildlife, on the design of the layout, design, adopted the "wall to wall day" will be wild animals graphic distortion on the window, meaning a cage of wild animals are people set up.
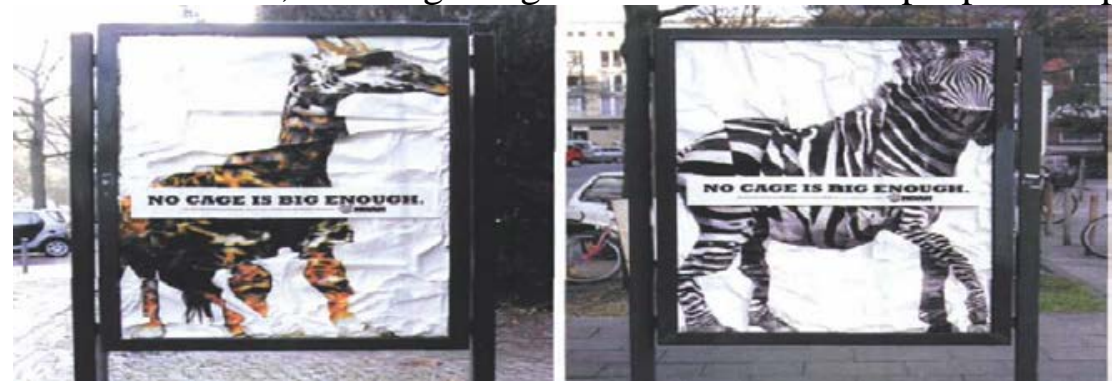

Fig.3 Wildlife protection poster design works of posters

In graphic design, the reasonable utilization of the transformation of visual space with simplified design elements, and enhance the visual effect of active images, cause the audience curiosity; To guide the visual process, convey information, enhance the aesthetic feeling of graphic design, and other effects. Visual space transformation is widely applied to advertising design, logo design, packaging design, graphic design creation such as plate design, through the, graphic and solid 
transformation, the transition between 2D and 3D, set, specific, objective and subjective image and abstract image and the conversion between reality and virtual form the full of mystery. In order to better use of the 3D visual space in the two-dimensional graphic space performance effect, Suggestions from 2 aspects:

(1) The use of representational space orientation, improve the occasion

In a limited amount of space, express movement and rhythm, with concise strokes by dot, line, face, silhouette image graphic design things, through the two-dimensional pattern varies with change, break through the two-dimensional planar space, reflect the beauty of nature from the movement of all things, with abstract expression of specific images.

(2) Use visual space continuity, with like

In two-dimensional design modeling adornment, more graphics to create the form of law to balance, symmetrical balance, asymmetrical balance, as the foundation, from the content to be performance, the final visual effect depends on the process of synthesis of visual space and art. Rules and irregular, simple and complex, symmetry and asymmetry, etc., are constructed with the theme, image, and cannot be isolated.

\section{Summary}

The development of the modern graphic design has increasingly depended on computer, the application of The virtual reality technology has changed the graphic design theory and method, runs through the whole process of the graphic design, from concept design to put on the market in the late prophase, The virtual reality technology can make the whole process of digital graphic design. Using the virtual reality technology, it can greatly shorten the graphic design process, improve efficiency, and reduce risk. With the rapid development of The virtual reality technology, the whole process of graphic design will be more and more dependent on The virtual reality technology, the development of product design in the future will be more quickly, more efficient.

\section{References}

[1] Ronald Azuma.A survey of augmented reality. Presence: Teleoperators and Virtual Environments. 2012

[2] Ronald T.Azuma,Yohan Baillot,Reinhold Behringer,et al.Recent advances in augmented reality. IEEE Computer Graphics and Applications. 2011

[3] Milgram, Paul,Kishino, Fumio.Taxonomy of mixed reality visual displays. IEICE Transactions on Information and Systems. 2012

[4] Yuan Yao,Miaoliang Zhu,Yunliang Jiang,and Guang Lu.A bare hand controlled AR map navigation system. Systems, Man and Cybernetics, 2004 IEEE International Conference. 2011

[5] G. Albertelli,R. A. Crawfis.Efficient subdivision of finite-element datasets into consistent tetrahedral. IEEE Visualization, Phoenix, AZ. 2013. 